\title{
Brainstem involvement and respiratory failure in COVID-19
}

\author{
Fiore Manganelli ${ }^{1}$ (D) Maria Vargas $^{1} \cdot$ Aniello lovino $^{1} \cdot$ Carmine lacovazzo $^{1} \cdot$ Lucio Santoro $^{1} \cdot$ Giuseppe Servillo $^{1}$
}

Received: 4 May 2020 / Accepted: 21 May 2020 / Published online: 29 May 2020

(C) Fondazione Società Italiana di Neurologia 2020

\begin{abstract}
Respiratory failure is the most worrisome problem of COVID-19. Patients may develop severe pneumonia requiring invasive mechanical ventilation and a significant proportion of them dies. It has been suggested that brainstem might play a role in severe respiratory failure of COVID-19 patients. We described three COVID-19 patients in ICU at Federico II Hospital in Naples that, although had recovered from pneumonia, could not be weaned from invasive mechanical ventilation. Our clinical evaluation was consistent with an involvement of the brainstem and especially of respiratory centre thus possibly explaining the weaning failure in patients that were awake and had recovered from lung involvement. Our data, though limited, indicate that brainstem involvement may play a role in respiratory failure and perhaps in the high death rate of COVID-19 patients. Moreover, the weaning failure from mechanical ventilation due to central respiratory drive depression might underlie the unusual long stay in ICU reported for COVID-19 patients.
\end{abstract}

Keywords Covid-19 $\cdot$ SARS-CoV-2 $\cdot$ respiratory failure $\cdot$ brainstem involvement

Respiratory failure is the most worrisome problem of COVID19. Patients may develop severe pneumonia requiring invasive mechanical ventilation and a significant proportion of them dies. Moreover, many patients fail early attempts at weaning and the time of intensive care unit (ICU) stay appears to be long [1-3].

It has been suggested that brainstem might play a role in severe respiratory failure of COVID-19 patients [3]. This hypothesis comes from animal models infected with other coronaviruses that have shown the brainstem to be severely affected and especially the respiratory centre (i.e. nucleus of solitary tract in the medulla oblongata) [4]. Under these premises, we evaluated three COVID-19 patients in ICU at Federico II Hospital in Naples that, although had recovered from pneumonia, could not be weaned from invasive mechanical ventilation due to depression of central respiratory drive.

At time of evaluation patient \#1 (male, 66 years, nasopharyngeal swab still positive) was intubated for 18 days and sedation was interrupted for 6 days, patient \#2 (female,

Fiore Manganelli and Maria Vargas equally contributed to this work Lucio Santoro and Giuseppe Servillo are senior authors

Fiore Manganelli

fiore.manganelli@unina.it; fioremanganelli@gmail.com

1 Department of Neurosciences, Reproductive Sciences and Odontostomatology, University of Naples Federico II, Naples, Italy
47 years, nasopharyngeal swab still positive) was intubated for 22 days and sedation was interrupted for 6 days, patient \#3 (female, 67 years, nasopharyngeal swab still positive) was intubated for 13 days and sedation was interrupted for 4 days.

Patients \#1 and \#2 opened eyes spontaneously but awareness was markedly reduced and they were able to perform only simple commands to verbal stimulus (e.g. to close the eyes both, to protrude the tongue only \#2). Painful stimulus to body and face elicited facial grimace. Pupils were equal and reactive to light. Corneal, oculocephalic, oculovestibular and cough reflexes were absent. Stopping mechanical ventilation did not trigger spontaneous breathing. Electroencephalogram (EEG) showed generalized slow activity. Brain MRI with contrast was normal (Fig. 1). These two patients died respectively 6 and 4 days after neurological evaluation.

Patient \#3 opened eyes spontaneously but she was not able to perform any simple commands. Painful stimulus to body and face elicited facial grimace. Pupils were equal and reactive to light. Corneal, oculocephalic, oculovestibular and cough reflexes were preserved. Stopping mechanical ventilation triggered spontaneous breathing even though it was not enough for weaning mechanical ventilator support. EEG showed generalized slow activity. Brain TC revealed only punctiform gliotic foci in right pons.

There is growing evidence of central nervous system (CNS) involvement in COVID-19 [5, 6] and our clinical findings are certainly consistent with an involvement of the brainstem and especially of respiratory centre. 
Fig. 1 Brain MRI sagittal FLAIR (a, c) and coronal T2-weighted (b, d) images from patient \#1 (a, b) and \#2 (c, d). No structural changes are visible
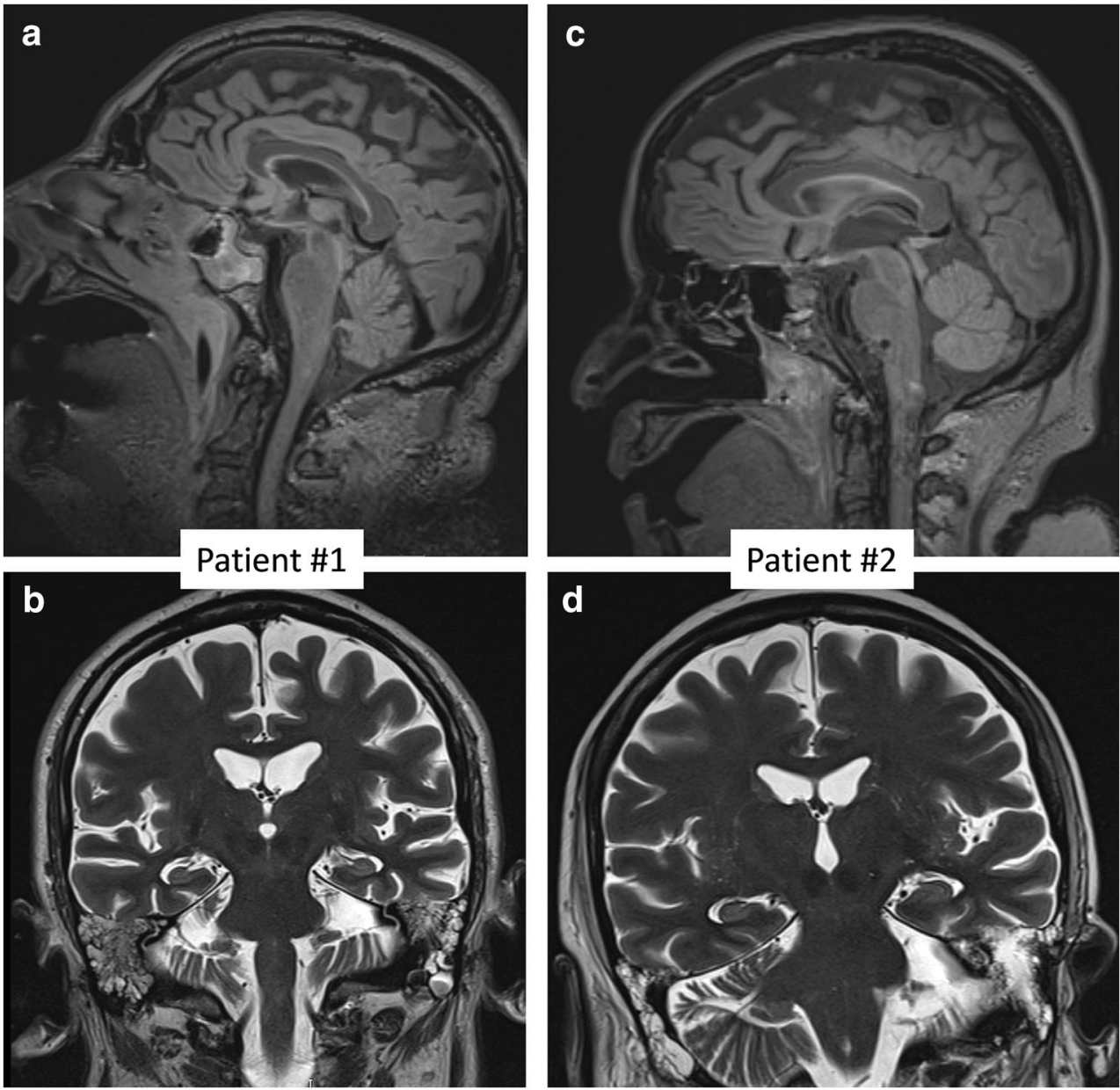

Thus, the impairment of this latter might explain the weaning failure from mechanical ventilation in our patients that were awake and had recovered from pneumonia. Nevertheless, the interpretation of clinical picture struggles with the lacking of brain structural radiological changes.

Though we do not have a definite explanation, we could figure that brainstem dysfunction may be related to centrally spreading of SARS-Cov-2 along the vagus nerve. This hypothesis was recently brought up by Tassorelli and colleagues [3] from the front-line of COVID-19 in Italy (Policlinico San Matteo of Pavia in Lombardy).

Really, experimental studies suggested that SARS-CoV and MERS-CoV as well other human coronaviruses could enter the CNS via olfactory nerves or vagus nerve that carries, to solitary nucleus in brainstem, visceral sensation from respiratory airways and lungs and taste sensation from the epiglottis and root of the tongue [4]. Of interest, a large proportion of COVID-19 patients precociously complains of anosmia and ageusia [7].

Thus, it is conceivable that also SARS-CoV-2, sharing a high degree of structural similarities with SARS-CoV and MERS-CoV, may enter CNS via cranial nerves.
In conclusion, our data, though limited, indicate that brainstem involvement may play a role in respiratory failure [4] and perhaps in the high death rate of COVID-19 patients. Just the two patients with more severe brainstem involvement died.

Moreover, the weaning failure from mechanical ventilation due to central respiratory drive depression might underlie the unusual long stay in ICU reported for COVID-19 patients [2].

Lastly, once the tremendous wave of the epidemic that is overwhelming ICUs will subside, a new scenario could manifest enclosing the prognosis and management of patients needing long-term mechanical ventilation.

Funding information No sources of funding or payment were received for this article.

Availability of data and materials Not applicable.

\section{Compliance with ethical standards}

Conflict of interest The authors have no competing interests.

Ethical approval None. 


\section{References}

1. Huang C, Wang Y, Li X, Ren L, Zhao J, Hu Y, Zhang L, Fan G, Xu J, Gu X, Cheng Z, Yu T, Xia J, Wei Y, Wu W, Xie X, Yin W, Li H, Liu M, Xiao Y, Gao H, Guo L, Xie J, Wang G, Jiang R, Gao Z, Jin Q, Wang J, Cao B (2020) Clinical features of patients infected with 2019 novel coronavirus in Wuhan, China. Lancet 395:497-506

2. Grasselli G, Zangrillo A, Zanella A, Antonelli M, Cabrini L, Castelli A, Cereda D, Coluccello A, Foti G, Fumagalli R, Iotti G, Latronico N, Lorini L, Merler S, Natalini G, Piatti A, Ranieri MV, Scandroglio AM, Storti E, Cecconi M, Pesenti A, for the COVID-19 Lombardy ICU Network (2020) Baseline characteristics and outcomes of 1591 patients infected with SARS-CoV-2 admitted to ICUs of the Lombardy region. Italy JAMA 323:1574. https://doi.org/10.1001/ jama.2020.5394

3. Tassorelli C, Mojoli F, Baldanti F, Bruno R, Benazzo M (2020 Apr 25) COVID-19: what if the brain had a role in causing the deaths? Eur J Neurol. https://doi.org/10.1111/ene.14275

4. Li YC, Bai WZ, Hashikawa T (2020) The neuroinvasive potential of SARS-CoV2 may play a role in the respiratory failure of COVID-19 patients. J Med Virol 92:552-555. https://doi.org/10.1002/jmv. 25728

5. Mao L, Jin H, Wang M, Hu Y, Chen $\mathrm{S}$, He Q, Chang J, Hong C, Zhou Y, Wang D, Miao X, Li Y, Hu B (2020) Neurologic manifestations of hospitalized patients with coronavirus disease 2019 in Wuhan, China. JAMA Neurol. https://doi.org/10.1001/jamaneurol. 2020.1127

6. Wu Y, Xu X, Chen Z, Duan J et al (2020) Nervous system involvement after infection with COVID-19 and other coronaviruses. Brain Behav Immun S0889-1591(20):30357-30353. https://doi.org/10. 1016/j.bbi.2020.03.031

7. Lechien JR, Chiesa-Estomba CM, De Siati DR et al (2020) Olfactory and gustatory dysfunctions as a clinical presentation of mild-tomoderate forms of the coronavirus disease (COVID-19): a multicenter European study. Eur Arch Otorhinolaryngol. https://doi.org/10. 1007/s00405-020-05965-1

Publisher's note Springer Nature remains neutral with regard to jurisdictional claims in published maps and institutional affiliations. 